\title{
自适应模糊控制在抑制海上漂浮式风力机 振动响应中的应用
}

\author{
杨佳佳，贺尔铭，舒俊成
}

(西北工业大学 航空学院, 陕西 西安 710072)

\begin{abstract}
摘 要: 海上漂浮式风力机是一个复杂的刚一柔耦合非线性系统, 难以建立其精确的动力学模型, 无法 利用常规控制方式提高风力机的抗风浪千扰能力。为解决此问题, 采用自适应模糊控制器 (AFC) 抑 制漂浮式风力机的动力响应。引入 2 个修正因子对模糊规则进行优选, 得到了传统模糊控制器 (FC) ; 为应对漂浮式风力机工作过程中平衡位置变化和结构参数摄动情况,在 FC 基础上增加了自适 应模块, 设计了一种 AFC, 并对其有效性进行了验证; 在随机风浪扰动和叶片桨距控制系统耦合作用 下, 研究了不同控制策略对海上漂浮式风力机振动响应的抑制效果。仿真结果表明, 目标值改变时, AFC 对目标值的跟踪能力明显优于 FC; 随机风浪扰动作用下, 相对于被动控制策略, AFC 对平台俯仰 (PFPI) 运动功率谱密度(PSD) 峰值的抑制效果可提高 $39 \%$ 。
\end{abstract}

\section{关 键 词: 海上漂浮式风力机; 振动抑制; 修正因子;模糊规则; 自适应模糊控制}

\section{中图分类号: 0328}

文献标志码: A
文章编号: 1000-2758(2021)02-0241-08
海上风能具有风速高和风切变小等优势, 已成 为最具开发价值的绿色能源之一 ${ }^{[1]}$ 。作为远深海 风能利用的重要载体, 海上漂浮式风力机是一个复 杂的风浪扰动-叶片桨距控制器-结构耦合的非线 性系统。在随机风浪作用下, 浮动平台、塔架和叶片 等结构将产生巨大的振动响应, 严重影响海上漂浮 式风力机的结构可靠性。因此, 采用不同控制方式 提高海上漂浮式风力机的抗风浪干扰能力, 抑制结 构的振动响应, 已成为当前海上漂浮式风力机研究 的重要课题。

被动控制策略是提高海上漂浮式风力机抗风浪 干扰能力的一种有效途径。2011 年, Lackner 等 ${ }^{[2]}$ 首次利用质量调谐阻尼器( TMD) 系统降低随机风 浪对风力机振动的影响, 并将机舱 TMD 模块集成到 风力机振动特性分析软件 FAST 中。随后, Jin、 $\mathrm{He}$ 等 ${ }^{\left[{ }^{4-4}\right]}$ 采用改进的人工鱼群算法 (AFSA) 优化了风 力机 TMD 参数, 结果表明, 最优 TMD 能够显著降低 风力机的塔顶前后 (TTFA) 挠度。2020 年, Zhang ${ }^{[5]}$ 建立了旋转叶片-TMD 的 2-自由度 (2-DOF) 动力学
模型, 根据频率调谐公式, 得到 TMD 的最优阻尼比, 发现 TMD 的最优阻尼比取决于叶轮的旋转速度。 Jahangiri 等 ${ }^{[6]}$ 采用三维摆调谐质量阻尼器 (3DPTMD) 和双线性冲击调谐质量阻尼器 (2PTMDs) 减 小 Spar 式风力机在风浪荷载作用下的三维振动, 结 果证明, 二者均能有效降低风力机的振动响应, 且 2 PTMDs 的行程约为 3D-PTMD 的 50\%。

近几年, 采用主动控制策略减小风力机结构振 动逐渐成为一个研究热点。Horacio 等 ${ }^{[7]}$ 基于主动 干扰抑制 (ADR) 的控制方式减小风力机的变频周 期性负荷扰动, 发现和被动控制相比, ADR 可以进 一步降低周期性负荷扰动对风力机结构振动的影 响。Jaime 等 ${ }^{[8]}$ 设计了一种基于叶尖挠度测量和单 桨距控制的新型叶片桨距主动控制器, 仿真结果证 明, 新型主动控制器可以降低叶轮关键部件的疲劳 损伤。Fitzgerald 等 $^{[9]}$ 结合漂浮式风力机的结构动 力学模型和概率评估工具, 研究了主动调谐质量阻 尼器 (ATMD) 对风力机塔架可靠性的影响, 分析发 现, 在额定风速下,ATMD 在一定程度上改善了塔架 
的可靠性。

2015 年以来, 本课题组 ${ }^{[10-12]}$ 基于拉格朗日能量 法建立了 Barge 式风力机的结构动力学模型, 并分 别在机舱和平台中配置 TMD, 结果表明, 机舱 TMD 和平台 TMD 均可有效提高结构的抗风浪干扰能力。 另外, 课题组研究了线性二次型调节器 (LQR) 对 Barge 式风力机振动响应的影响, 发现和机舱 TMD 系统相比, LQR 控制器的减振效果可提升 $20 \%$ 左右 ${ }^{[13-14]}$ 。

常规最优控制策略, 如 LQR 控制、H 控制等依 赖于精确的动力学模型。海上漂浮式风力机是一个 复杂的刚-柔耦合非线性系统, 在随机风浪引起的 风力机振动平衡位置变化、叶片桨距控制器耦合效 应导致的结构参数摄动、恶劣的海洋环境引起的结 构腐蚀和疲劳损伤等多因素作用下, 风力机动态特 性难以掌握, 建立精确的动力学模型非常困难。此 时, 采用常规最优控制策略减小随机风浪对风力机 结构振动的影响是不可行的。模糊控制具有不依赖 于精确的动力学模型、鲁棒性好、抗干扰能力强等优 点。因此, 有必要在随机风浪扰动和叶片桨距控制 器耦合作用下, 进一步研究模糊控制策略对海上漂 浮式风力机结构振动的影响。本文以美国 NREL 实 验室开发的 Spar 型漂浮式风力机模型为基础, 设计 开发了自适应模糊控制器, 在随机风浪扰动和叶片 桨距控制器耦合作用下, 研究了不同控制策略对 Spar 风力机结构振动的控制效果。

\section{Spar 式风力机刚一柔耦合非线性动 力学模型}

Spar 式风力机刚-柔耦合结构简图见图 1a), 平 台为刚体, 塔架和叶片为柔性体, 机舱为固定于塔顶 的质点。风力机浮式平台通过针线系统和海床相 连, 针线系统简化为平台对应 DOF 的弹簧, 随机风 浪沿 $X$ 轴作用于叶轮和浮式平台。 $O X Y Z$ 为惯性坐 标系, $O X Y$ 平面与静水面平行, $Z$ 轴坚直向上。 $o^{h} i j k$ 为固定于叶轮上的旋转坐标系, 原点 $o$ 固定于轮鈠, $i$ 轴沿叶片 1 长度方向, $j$ 轴在旋转平面内垂直于 $i$ 轴, $k$ 轴垂直于 $o^{h} i j$ 平面。Spar 式风力机的基本参数 见表 1 。
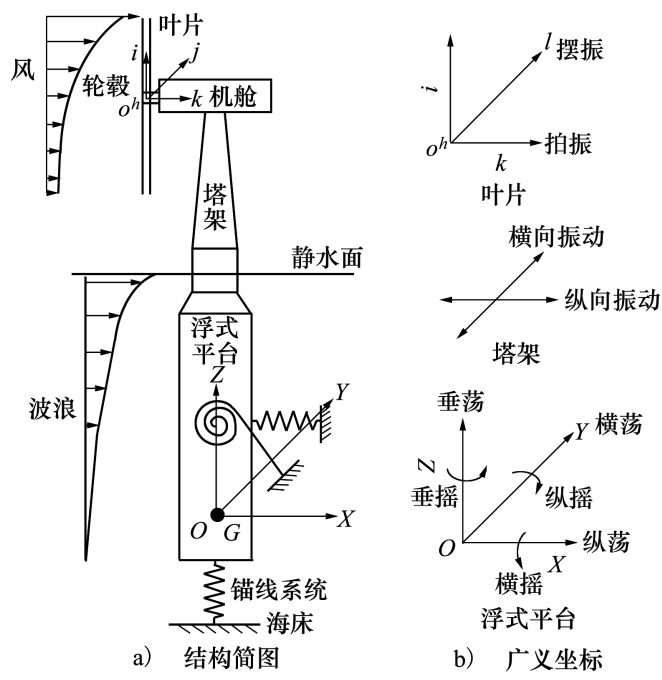

塔架

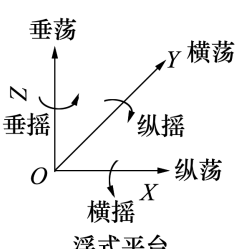

浮式平台

b) 广义坐标

图 1 Spar 式风力机结构简图和 DOF

表 1 Spar 式风力机的基本参数

参数 值

额定功率/MW

5

切人、额定、切出风速 $/\left(\mathrm{m} \cdot \mathrm{s}^{-1}\right)$

$3,11.4,25$

叶片数

3

叶轮, 轮毂直径 $/ m$

126,3

叶轮质量 $/ \mathrm{kg}$

110000

机舱质量 $/ \mathrm{kg}$

240000

塔架质量 $/ \mathrm{kg}$

347460

平台质量 $($ 含压舱物) $/ \mathrm{kg}$

7466330

平台直径

雉体以上 $6.5 \mathrm{~m}$, 雉体以下 $9.4 \mathrm{~m}$

平台长度

SWL 至雉顶 $4 \mathrm{~m}$, 至雉底 $12 \mathrm{~m}$, 至平台底部 $120 \mathrm{~m}$

机舱尺寸

$24 \mathrm{~m} \times 8 \mathrm{~m} \times 6 \mathrm{~m}$

$\mathrm{SWL}$ 到轮鈠中心的距离 $/ \mathrm{m}$

90

$\mathrm{SWL}$ 到塔顶的距离 $/ \mathrm{m}$

87.6

$\mathrm{SWL}$ 到平台重心的距离 $/ \mathrm{m}$

89.9

在浮式平台和机舱分别安装 TMD 系统, 如图 2 所示。 $M_{\mathrm{p}}$ 和 $M_{\mathrm{n}}$ 分别为平台 / 机舱 TMD 的质量块, 则 TMD 系统由质量 $m$ 、刚度系数 $k$ 和阻尼系数 $c$ 组成。 另外, 在机舱中安装主动作动器 $f^{\mathrm{Tn}}$, 对 $M_{\mathrm{n}}$ 施加主动 控制力 $u$, 即可构成混合质量阻尼器( HMD) 系统。 


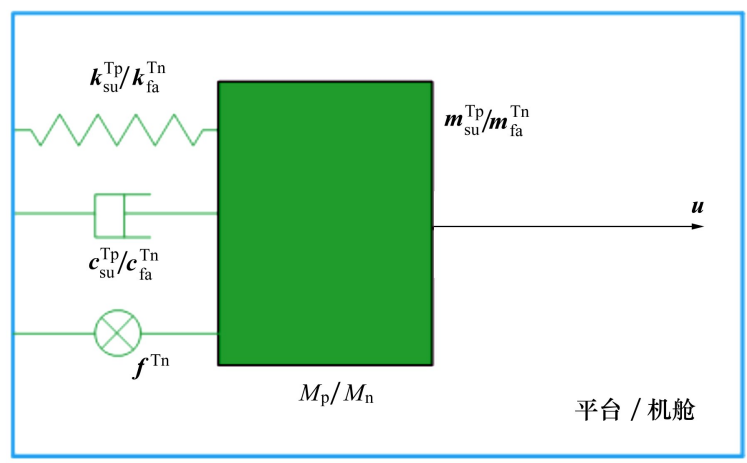

图 2 平台/机舱 TMD 系统示意图

最优 TMD 参数如表 2 所示。

表 2 最优 TMD 参数

\begin{tabular}{cccc}
\hline 模块 & 质量 $/ \mathrm{kg}$ & 刚度 $/\left(\mathrm{N} \cdot \mathrm{m}^{-1}\right)$ & 阻尼 $/\left(\mathrm{Ns}_{\mathrm{s}} \cdot \mathrm{m}^{-1}\right)$ \\
\hline 平台 TMD & $3.988 \times 10^{5}$ & $6.004 \times 10^{4}$ & $2.895 \times 10^{5}$ \\
机舱 TMD & $1.047 \times 10^{4}$ & $8.666 \times 10^{4}$ & $4.203 \times 10^{3}$ \\
\hline
\end{tabular}

设 Spar 式风力机的广义坐标向量 $\boldsymbol{q}$ 为

$$
\begin{aligned}
& {\left[\begin{array}{l}
\boldsymbol{q}=\left[\begin{array}{llll}
\boldsymbol{q}^{\mathrm{p}} & \boldsymbol{q}^{\mathrm{t}} & \boldsymbol{q}^{\mathrm{b}} & \boldsymbol{q}^{\mathrm{T}}
\end{array}\right]^{\mathrm{T}} \\
\boldsymbol{q}^{\mathrm{p}}=\left[\begin{array}{llllll}
q_{s u}^{\mathrm{p}} & q_{s w}^{\mathrm{p}} & q_{h e}^{\mathrm{p}} & q_{r o}^{\mathrm{p}} & q_{p i}^{\mathrm{p}} & q_{y a}^{\mathrm{p}}
\end{array}\right]^{\mathrm{T}}
\end{array}\right.} \\
& \left\{\boldsymbol{q}^{\mathrm{t}}=\left[\begin{array}{ll}
q_{f a}^{\mathrm{t}} & q_{s s}^{\mathrm{t}}
\end{array}\right]^{\mathrm{T}}\right. \\
& \boldsymbol{q}^{\mathrm{b}}=\left[\begin{array}{ll}
q_{f l}^{\mathrm{b}_{a}} & q_{e d}^{\mathrm{b}_{a}}
\end{array}\right]^{\mathrm{T}} \\
& \boldsymbol{q}^{\mathrm{T}}=\left[\begin{array}{ll}
q_{s u}^{\mathrm{T}_{\mathrm{p}}} & q_{f a}^{\mathrm{T}_{\mathrm{n}}}
\end{array}\right]^{\mathrm{T}}
\end{aligned}
$$

式中:上标 $\mathrm{p}, \mathrm{t}, \mathrm{b}, \mathrm{T}$ 分别表示平台、塔架、叶片和 $\mathrm{TMD}$, 上标 $\mathrm{b}_{a}$ 分别代表 3 个叶片 $(a=1,2,3) ; \mathrm{T}_{\mathrm{p}}, \mathrm{T}_{\mathrm{n}}$ 表示平台 TMD 和机舱 TMD, 下标为对应广义坐标的 缩写, 详见图 $1 \mathrm{~b})$, 本文假定 $\boldsymbol{q}$ 完全可测。

那么, Spar 式风力机刚一柔耦合非线性动力学 模型可记为

$$
\ddot{\boldsymbol{q}}=\boldsymbol{f}(\boldsymbol{q}, \dot{\boldsymbol{q}})+\boldsymbol{g}(\boldsymbol{q}, \dot{\boldsymbol{q}}) \boldsymbol{u}+\boldsymbol{f}_{d}
$$

式中: $f(\cdot)$ 和 $g(\cdot)$ 为未知有界的非线性函数; $f(\cdot)$ 代表风力机的结构参数; $\boldsymbol{g}(\cdot)$ 表示控制力的位置矩 阵; $\boldsymbol{f}_{d}$ 为随机风浪扰动及结构参数摄动。

通过文献 $[14]$ 的研究发现, 抑制 PFPI 运动不 仅可以降低风力机平台的振动响应, 还可减小 TTFA 的低频振动, 因此, PFPI 运动是表征风力机振动响 应的一个关键参数。本文将 PFPI 作为控制对象, 通过计算 PFPI 运动和目标值之间的误差与误差变 化率实现模糊控制。

\section{2 传统模糊控制器设计}

图 3 为 Spar 式风力机模糊控制系统。 $y_{m}$ 为目标 值, 即风力机在随机风浪作用下的振动平衡位置; $e$ 和 $e_{c}$ 分别代表误差与误差变化率, $e=y_{m}-q, e_{c}=\dot{e}_{\text {。 }}$ $u$ 为控制器输出的控制力; $K_{e}, K_{e_{c}}$ 为量化因子, $K_{u}$ 表 示比例因子。

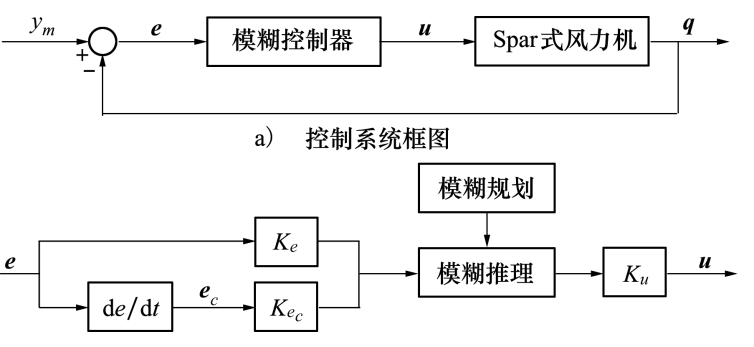

b) 模糊控制器框图

图 3 模糊控制系统

模糊规则是模糊控制器的核心, 其基本形式 如下

$$
\text { 如果 } \boldsymbol{e}=\boldsymbol{E} \text { 且 } e_{c}=\boldsymbol{E}_{C} \text {, 则 } \boldsymbol{u}=\boldsymbol{U}
$$

式中, $\boldsymbol{E}, \boldsymbol{E}_{C}, \boldsymbol{U}$ 分别代表 $e, e_{c}$ 和 $\boldsymbol{u}$ 的模糊子集。

模糊规则直接影响模糊控制效果的优劣, 为了 减小设计者主观经验对模糊规则的影响, 针对 Spar 式风力机复杂的工作环境, 在文献 [14] 中所推导的 线性数学模型基础上, 以 PFPI 标准差最小为优化 目标, 引人 2 个修正因子, 优选出相应的模糊规则。 具体过程为:

1) 将 $\boldsymbol{e}, e_{c}$ 和 $\boldsymbol{u}$ 的论域划分为 7 个模糊子集, 分 别用 -3 (负大) 、 -2 (负中) 、 -1 (负小)、 0 (零) 、 1 (正 小)、2(正中)、3(正大) 表示。

2) 引人修正因子, 利用公式 (4), 得到多个输 出模糊子集。

$$
\boldsymbol{U}=\left\{\begin{array}{l}
\left\langle\alpha_{1} \times E+\left(1-\alpha_{1}\right) \times E_{C}\right\rangle, E= \pm 1,0 \\
\left\langle\alpha_{2} \times E+\left(1-\alpha_{2}\right) \times E_{C}\right\rangle, E= \pm 2, \pm 3
\end{array}\right.
$$

式中, $\alpha_{1}, \alpha_{2}$ 表示修正因子, 取值范围为 $[0,1] ;\langle\cdot\rangle$ 表示四舍五人取整。

3) 将不同的模糊子集嵌人图 3b) 中,结合文献 [14] 给出的数学模型, 计算 PFPI 标准差。

图 4 为工况 1 和工况 3 的 PFPI 标准差随 $\alpha_{1}$, $\alpha_{2}$ 变化规律。可以看出, 工况 1 中, $\alpha_{1}$ 和 $\alpha_{2}$ 分别取 $0.5,0.6$ 时, 标准差最小;工况 3 中, $\alpha_{1}, \alpha_{2}$ 分别取 0.5 , 0.4 时,PFPI 的标准差最小。 

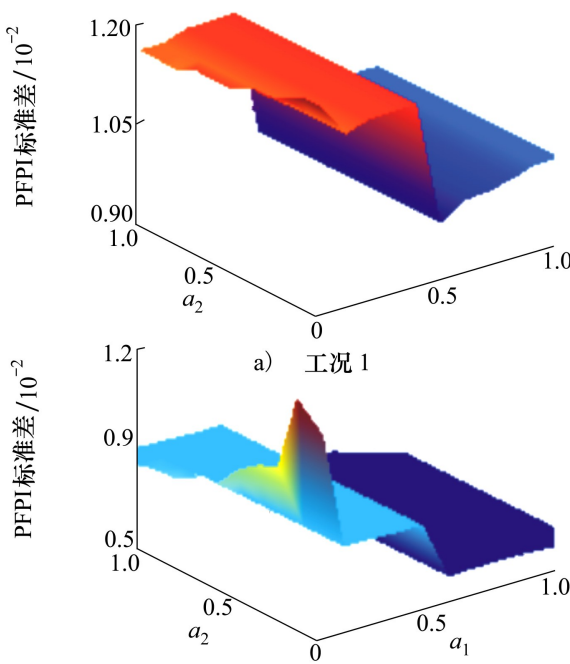

b) 工况 3

图 4 PFPI 标准差随 $\alpha_{1}, \alpha_{2}$ 的变化规律

使用乘积推理机、单值模糊器和中心平均解模 糊器, 得到 $\mathrm{FC}$ 为

$$
\left\{\begin{array}{l}
\boldsymbol{u}=\boldsymbol{\theta}^{\mathrm{T}} \boldsymbol{\xi}(\boldsymbol{q}) \\
\boldsymbol{\theta}=\left[y^{11}, y^{12}, \cdots, y^{21}, y^{22}, \cdots, y^{77}\right]^{\mathrm{T}} \\
\boldsymbol{\xi}(\boldsymbol{q})=\frac{\prod_{i=1}^{2} \mu_{i}^{l i}\left(x_{i}\right)}{\sum_{l_{1}=1}^{7} \sum_{l_{2}=1}^{7}\left(\prod_{i=1}^{2} \mu\left(x_{i}\right)\right)}
\end{array}\right.
$$

式中: $y$ 为模糊规则中 $U$ 对应的模糊数; $\boldsymbol{\theta}$ 表示 49 条 模糊规则中 $U$ 对应的模糊数组成的向量, 共包含 49 个元素; $l_{1}, l_{2}$ 分别表示 $\boldsymbol{E}$ 和 $E_{C}$ 模糊子集的编号; $l_{1}=$ $l_{2}=1,2, \cdots 7 ; x_{1}$ 和 $x_{2}$ 代表 $\boldsymbol{e}$ 和 $\boldsymbol{e}_{c}$ 的取值; $\mu$ 为 $x_{1}$ 和 $x_{2}$ 对应的隶属度, 隶属度采用高斯隶属函数计算; $\boldsymbol{\xi}(\boldsymbol{q})$ 是一个含有 49 个元素的列向量, 表示每条模 糊规则输人值的隶属度之积除以 49 条规则的隶属 度之积的和。

\section{3 自适应模糊控制器设计}

假设传统模糊控制器的参数 $\boldsymbol{\theta}$ 可调, 则传统模 糊控制器进化为自适应模糊控制器。因此, 本节主 要根据 Lyapunov 第二方法设计自适应模糊控制器。

根据文献 $[15]$, 误差状态方程可写为

$$
\dot{\boldsymbol{e}}=\boldsymbol{\Lambda} \boldsymbol{e}+\boldsymbol{g}(\boldsymbol{q}, \boldsymbol{q})\left[u^{*}-\bar{u}\right]
$$

式中: $u^{*}$ 和 $\bar{u}$ 分别为理想控制器和 AFC 的控制力 输出;

$$
\boldsymbol{\Lambda}=\left[\begin{array}{ccccccc}
0 & 1 & 0 & 0 & \cdots & 0 & 0 \\
0 & 0 & 1 & 0 & \cdots & 0 & 0 \\
\vdots & \vdots & \vdots & \vdots & & \vdots & \vdots \\
0 & 0 & 0 & 0 & \cdots & 0 & 1 \\
-k_{n} & -k_{n-1} & \cdots & \cdots & \cdots & \cdots & -k_{1}
\end{array}\right]
$$

$\boldsymbol{k}=\left(\begin{array}{lll}k_{n} & \cdots & k_{1}\end{array}\right)^{\mathrm{T}}$ 使得多项式 $s^{n}+k_{1} s^{n-1}+\cdots+k_{n}$ 的所有根均在复平面的左半开平面上。

定义最小逼近误差 $w=\bar{u}-u^{*}$, 那么误差状态方 程可以改写为

$$
\dot{\boldsymbol{e}}=\boldsymbol{\Lambda} \boldsymbol{e}+\boldsymbol{g}(\boldsymbol{q}, \dot{\boldsymbol{q}})\left[\boldsymbol{\theta}^{*}-\overline{\boldsymbol{\theta}}\right] \boldsymbol{\zeta}(\boldsymbol{q})-\boldsymbol{g}(\boldsymbol{q}, \dot{\boldsymbol{q}}) w
$$

式中, $\boldsymbol{\theta}^{*}$ 为可调参数 $\overline{\boldsymbol{\theta}}$ 的理想值。

选择 Lyapunov 函数

$$
\boldsymbol{V}=\frac{1}{2} \boldsymbol{e}^{\mathrm{T}} \boldsymbol{P} \boldsymbol{e}+\frac{1}{2 \gamma_{\theta}}\left(\boldsymbol{\theta}^{*}-\overline{\boldsymbol{\theta}}\right)^{\mathrm{T}}\left(\boldsymbol{\theta}^{*}-\overline{\boldsymbol{\theta}}\right)
$$

式中, $\boldsymbol{P}$ 正定且满足 Lyapunov 方程 $(9) ; r_{\theta}$ 为正 常数。

$$
\boldsymbol{\Lambda}^{\mathrm{T}} \boldsymbol{P}+\boldsymbol{\Lambda P}=-\boldsymbol{Q}
$$

式中, $Q$ 为正定矩阵。

对 $\boldsymbol{V}$ 求导可得

$$
\begin{gathered}
\dot{\boldsymbol{V}}=-\frac{1}{2} \boldsymbol{e}^{\mathrm{T}} \boldsymbol{Q} \boldsymbol{e}+\frac{1}{2 \gamma_{\theta}}\left(\boldsymbol{\theta}^{*}-\overline{\boldsymbol{\theta}}\right)^{\mathrm{T}}\left[\gamma_{\theta} \boldsymbol{e}^{\mathrm{T}} \boldsymbol{P} \boldsymbol{\xi}(\boldsymbol{q})-\overline{\boldsymbol{\theta}}\right] \\
-\boldsymbol{e}^{\mathrm{T}} \boldsymbol{P} \boldsymbol{g}(\boldsymbol{q}, \dot{\boldsymbol{q}}) w
\end{gathered}
$$

选择可调参数 $\overline{\boldsymbol{\theta}}$ 的自适应律为

$$
\dot{\overline{\boldsymbol{\theta}}}=\gamma_{\theta} \boldsymbol{e}^{\mathrm{T}} \boldsymbol{P} \boldsymbol{\xi}(\boldsymbol{q})
$$

即可保证 $\dot{\boldsymbol{V}}=-\frac{1}{2} \boldsymbol{e}^{\mathrm{T}} \boldsymbol{Q e}-\boldsymbol{e}^{\mathrm{T}} \boldsymbol{P} \boldsymbol{g}(\boldsymbol{q}, \dot{\boldsymbol{q}}) w<0$ 。为保证 一般性,所有随机风浪工况对应的可调参数 $\overline{\boldsymbol{\theta}}$ 初值 均由 $\alpha_{1}=\alpha_{2}=0.5$ 确定。

由此可得 $\mathrm{AFC}$ 为

$$
\left\{\begin{array}{l}
\overline{\boldsymbol{u}}=\overline{\boldsymbol{\theta}}^{\mathrm{T}} \boldsymbol{\xi}(\boldsymbol{q}) \\
\overline{\boldsymbol{\theta}}=\sum_{l_{1}=1}^{7} \sum_{l_{2}=1}^{7} \overline{\boldsymbol{y}}^{l_{l} l_{2}}
\end{array}\right.
$$

\section{4 自适应模糊控制器有效性验证}

本节采用 2 种方法验证 AFC 的有效性:

1) 给定方波作为目标值, 研究 FC 和 AFC 作用 下 PFPI 的跟踪效果;

2) 结构存在参数摄动 $\boldsymbol{f}_{d}$ 时, 研究 $\mathrm{FC}$ 和 $\mathrm{AFC}$ 作 用下 PFPI 对方波的跟踪效果。

图 5a) 为目标值变化时, FC 与 $\mathrm{AFC}$ 对目标值的 
跟踪图, 明显的, AFC 能够更好更快地跟踪目标值。 图 5b) 为跟踪误差, 由于目标值突变, 导致跟踪误差 出现尖峰; 方波幅值为 2 时, FC 的最小跟踪误差为 $0.1243, \mathrm{AFC}$ 的最小跟踪误差为 0.0435 。图 5c) 为
控制力输出, 可知, 当跟踪误差出现尖峰时, 需要一 个更大的控制力使峰值快速下降, 此时, AFC 的控 制力输出约为 FC 的 1.89 倍; 当跟踪误差趋近于 0 时, 控制力逐渐平缓。

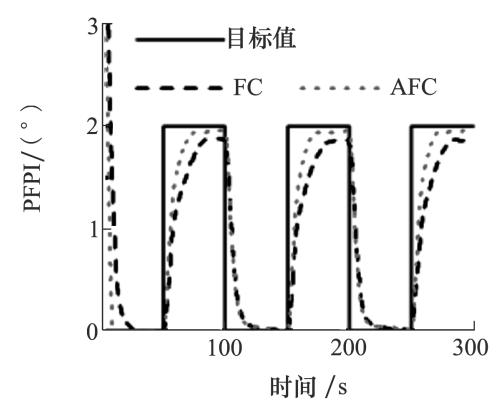

a) 跟踪图

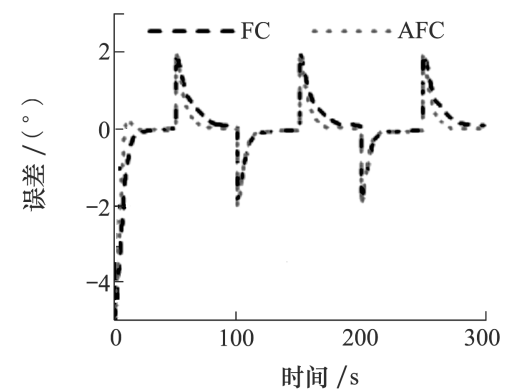

b) 跟踪误差

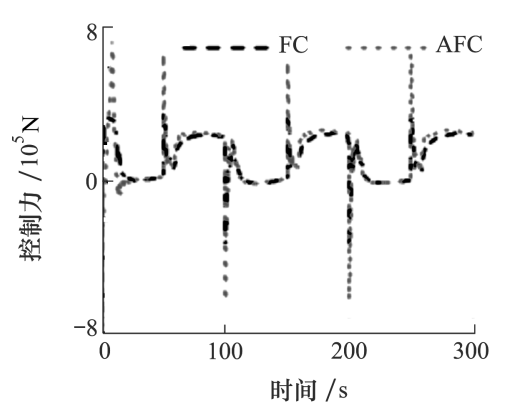

c) 控制力输出

图 5 PFPI 跟踪效果

图 6 为结构存在参数摄动 $f_{d}=3.6 \times 10^{8} \times \sin$ (1.047 1t) 时,PFPI 对目标值的跟踪效果。由于存 在参数摄动, 前 $30 \mathrm{~s}$ 的跟踪误差和控制力输出存在
较大波动, 随后达到一个相对平稳的跟踪过程。 $\mathrm{FC}$ 最小跟踪误差为 $0.126, \mathrm{AFC}$ 跟踪误差为 $0.055 ; \mathrm{AFC}$ 控制力输出峰值为 $\mathrm{FC}$ 峰值的 1.92 倍。
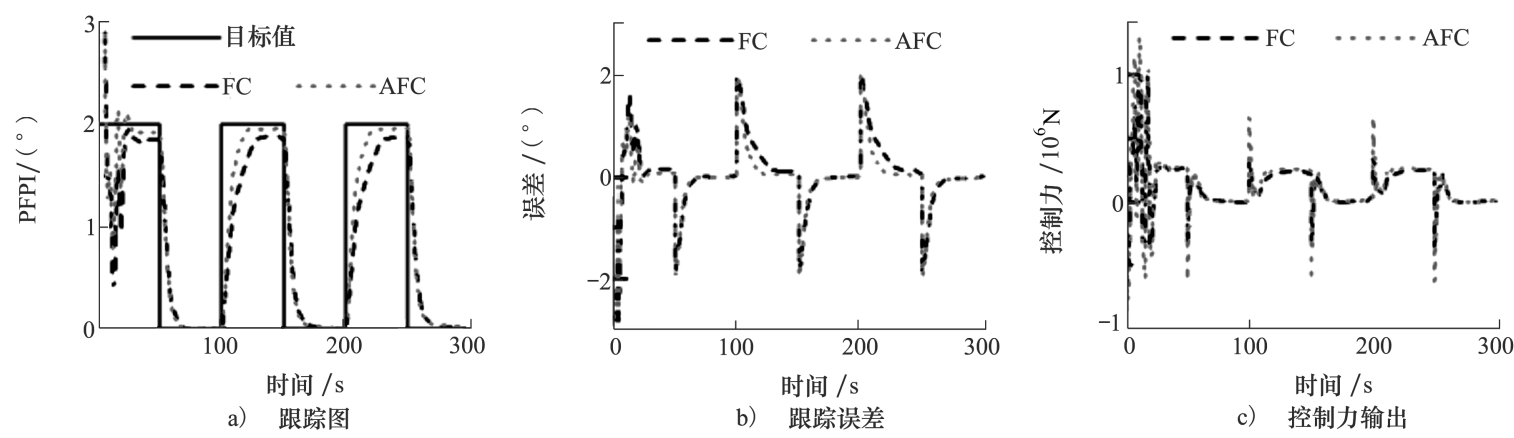

图 6 参数摄动时 PFPI 跟踪效果

由图 5 和图 6 可知, AFC 对目标值的跟踪能力 明显优于 FC, 因此,所设计的 AFC 是有效可行的。

\section{5 基于模糊控制的海上漂浮式风力机 振动抑制研究}

湍流风的风速和风压相对于时间是随机变化 的,波浪的波高、波长、方向、位相是由不同频率的随 机波叠加而成的, 导致很难准确预测海上风浪工况。 如果用轮毂处风速代替作用于风力机的湍流风, 将 波长和位相作为随机变量, 那么可用随机过程理论 反映海上波浪的随机特性。根据海上风力机设计标 准 IEC-3 的规定, 风速谱选择 Kaimal 谱, 波浪谱采 用 Pierson-Moskowitz 谱, 海上漂浮式风力机常用的
风浪工况基本参数见表 3 。

表 3 风浪工况基本参数

\begin{tabular}{ccccc}
\hline 工况 & $\begin{array}{c}\text { 轮毂平均风速/ } \\
\left(\mathrm{m} \cdot \mathrm{s}^{-1}\right)\end{array}$ & 湍流强度 & $\begin{array}{c}\text { 有效波高/ 波谱周期/ } \\
\mathrm{m}\end{array}$ & \begin{tabular}{c}
$\mathrm{s}$ \\
\hline 1
\end{tabular} \\
\hline & 8 & 0.20 & 2.0 & 12 \\
2 & 12 & 0.16 & 2.6 & 13 \\
3 & 18 & 0.15 & 4.0 & 15 \\
4 & 24 & 0.12 & 5.6 & 18 \\
\hline
\end{tabular}

通过 6 次 $300 \mathrm{~s}$ 的海上风浪模拟, 取其平均值, 则海上风浪时程曲线见图 7。可以看出, 风浪具有 很强的随机性。工况 1 风速较低, 均值约为 $8 \mathrm{~m} / \mathrm{s}$, 波高峰值约为 $1.47 \mathrm{~m}$; 工况 3 平均风速约为 $18 \mathrm{~m} / \mathrm{s}$; 波高峰值约为 $2.84 \mathrm{~m}$ 。 


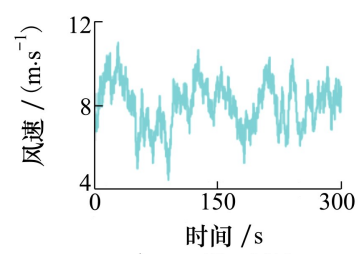

a) 工况 1 风速

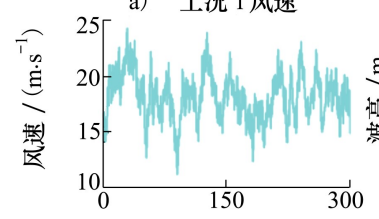

时间 /s

c）工况 3 风速

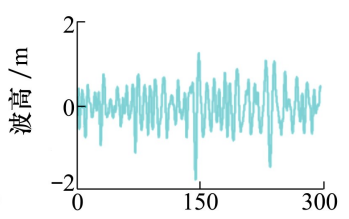

b） 时间 $/ \mathrm{s}$

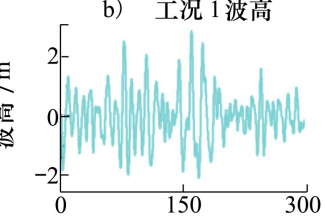

d） 时间 /s
图 7 海上风浪时程曲线

图 8 为随机风浪作用下, 叶片桨距角的时程曲 线。由图可知,工况 1 的风速较低, 叶片桨距控制系 统停止工作, 桨距角维持为 0 ; 工况 2 的桨距角变化 范围为 $0^{\circ} \sim 10^{\circ}$; 工况 3 和工况 4 的风速超出额定 值, 桨距角随风速的改变而变化, 且风速越大, 桨距 角越大。

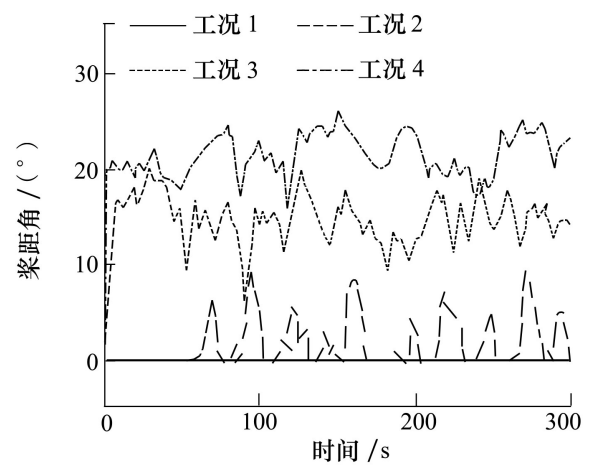

图 8 叶片桨距角变化时程曲线

图 9 为在随机风浪扰动和叶片桨距控制系统耦 合作用下, 不同控制方式的 PFPI 运动 PSD 图。明 显的, FC 和 AFC 的 PSD 峰值远远低于 TMD 被动控
制的峰值, 且 PSD 能量主要集中在 $0 \sim 0.15 \mathrm{~Hz}$ 内。 和被动控制相比, 工况 1 中, FC 和 AFC 对 PFPI 运 动的 PSD 峰值的抑制效果为 $34.24 \%, 38.97 \%$, 工况 3 中, 2 种模糊控制作用的 PSD 峰值分别减小了 $35.95 \%, 39.06 \%$ 。

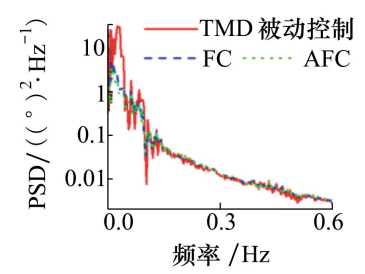

a) 工况 1

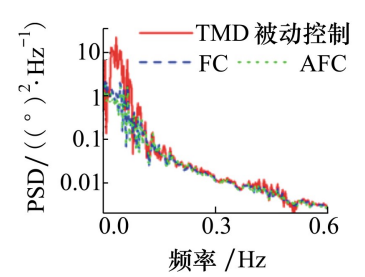

b) 工况 3
图 9 不同工况的 PFPI 运动 PSD

图 10 为 $\mathrm{FC}$ 和 $\mathrm{AFC}$ 的控制力 $\mathrm{PSD}$ 图。同样的, 控制力能量主要集中在 $0 \sim 0.15 \mathrm{~Hz}$ 内; 不同工况下, AFC 的控制力峰值约为 FC 峰值的 1.1 倍。

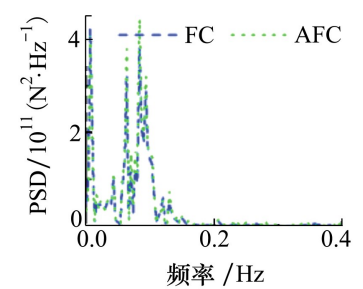

a) 工况 1

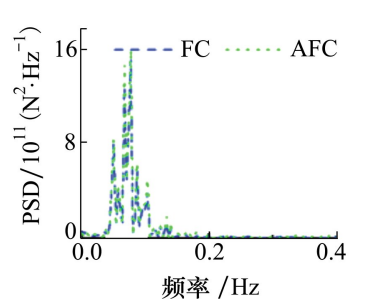

b) 工况 3
图 10 不同工况的控制力 PSD

表 4 为时域中不同模糊控制方式对随机风浪扰 动的抑制能力, 均值代表控制后平衡位置接近目标 位置的程度, 标准差反映振动响应的波动程度。 AFC 的均值抑制率小于 FC, 表明 AFC 作用下, PFPI 及 TTFA 更接近于 TMD 被动控制的均值 (目标位 置) ; AFC 的标准差抑制率高于 FC, 则 AFC 对 PFPI 运动和 TTFA 挠度的抑制能力更强。

表 4 时域中不同控制方式抑制风浪扰动的能力

\begin{tabular}{|c|c|c|c|c|c|c|c|c|}
\hline \multirow{3}{*}{ 控制方式 } & \multicolumn{4}{|c|}{ 工况 1 抑制率/\% } & \multicolumn{4}{|c|}{ 工况 2 抑制率/\% } \\
\hline & \multicolumn{2}{|c|}{ PFPI } & \multicolumn{2}{|c|}{ TTFA } & \multicolumn{2}{|c|}{ PFPI } & \multicolumn{2}{|c|}{ TTFA } \\
\hline & 均值 & 标准差 & 均值 & 标准差 & 均值 & 标准差 & 均值 & 标准差 \\
\hline $\mathrm{FC}$ & 2.527 & 58.136 & 0.958 & 22.670 & 1.033 & 54.492 & 0.385 & 17.367 \\
\hline \multirow[t]{2}{*}{$\mathrm{AFC}$} & 2.081 & 62.405 & 0.776 & 23.088 & 1.010 & 58.436 & 0.370 & 19.011 \\
\hline & \multicolumn{4}{|c|}{ ，工况 3 抑制率/\% } & \multicolumn{4}{|c|}{ 工况 4 抑制率/\% } \\
\hline \multirow[t]{2}{*}{ 控制方式 } & \multicolumn{2}{|c|}{ PFPI } & \multicolumn{2}{|c|}{ TTFA } & \multicolumn{2}{|c|}{ PFPI } & \multicolumn{2}{|c|}{ TTFA } \\
\hline & 均值 & 标准差 & 均值 & 标准差 & 均值 & 标准差 & 均值 & 标准差 \\
\hline $\mathrm{FC}$ & 2.122 & 51.811 & 0.886 & 22.313 & 1.321 & 44.777 & 0.381 & 13.219 \\
\hline $\mathrm{AFC}$ & 1.713 & 56.786 & 0.792 & 24.258 & 1.092 & 53.179 & 0.285 & 17.728 \\
\hline
\end{tabular}




\section{6 结 论}

本文基于 Lyapunov 第二方法设计了自适应模 糊控制器, 并研究了不同模糊控制策略抑制风力机 振动响应的能力。主要结论如下:

1) 相比于传统模糊控制器,所设计的自适应模 糊控制器能够更好更快地跟踪目标值; 跟踪误差出 现尖峰时, 控制力输出达到最大。
2) 结构存在参数摄动时, 前 $30 \mathrm{~s}$ 的跟踪误差和 控制力输出存在较大波动, 随后的跟踪过程相对 平稳。

3) 叶片桨距控制器耦合作用下, 相对于 TMD 被动控制策略, 自适应模糊控制器对平台俯仰运动 的 PSD 峰值的最大抑制效果为 $39 \%$ 左右。

4) 不同工况下, 自适应模糊控制器的控制力峰 值约为传统模糊控制器峰值的 1.1 倍, 且控制力能 量主要集中在 $0 \sim 0.15 \mathrm{~Hz}$ 内。

\section{参考文献:}

[1] KALDELLIS J K, KAPSALI M, KATSANOU E. Renewable energy applications in Greece-what is the public attitude? [J]. Energy Policy, 2012, 42: 37-48

[2] LACKNER M A, ROTEA M A. Passive structural control of offshore wind turbines [J]. Wind energy, 2011, 14(3): 373-388

[3] JIN X, XIE S, HE J, et al. Optimization of tuned mass damper parameters for floating wind turbines by using the artificial fish swarm algorithm $[\mathrm{J}]$. Ocean engineering, 2018, 167: 130-141

[4] HE J, JIN X, XIE S, et al. Multi-body dynamics modeling and TMD optimization based on the improved AFSA for floating wind turbines $[J]$. Renewable Energy, 2019, 141: 305-321

[5] ZHANG Z L. Optimal tuning of the tuned mass damper(TMD) for rotating wind turbine blades [J]. Engineering Structures, 2020, 207: 110209

[6] JAHANGIRI V, SUN C. Three-dimensional vibration control of offshore floating wind turbines using multiple tuned mass dampers $[\mathrm{J}]$. Ocean Engineering, 2020, 206: 107196

[7] HORACIO C E, JOHN C R, SERGIO A. Rejection of varying-frequency periodic load disturbances in wind-turbines through active disturbance rejection-based control $[\mathrm{J}]$. Renewable Energy, 2019, 141: 217-235

[8] JAIME L, WAI H L, ALBERT M U, et al. Active tip deflection control for wind turbines[ J]. Renewable Energy, 2020, 149: $445-454$

[9] FITZGERALD B, SARKAR S, STAINO A. Improved reliability of wind turbine towers with active tuned mass dampers (ATMDs) [J]. Journal of Sound and Vibration, 2018, 419: 103-122

[ 10] HU Y,HE E,ZHANG Y. Optimization design of TMD for vibration suppression of offshore floating wind turbine $[\mathrm{J}]$. International Journal of Plant Engineering and Management, 2015, 1(20): 13-27

[11] 贺尔铭, 张扬, 胡亚琪. 3 种典型海上浮动式风机动力学特性比较分析 $[\mathrm{J}]$. 太阳能学报, 2015, 36(12): 2874-2881

HE Erming, ZHANG Yang, HU Yaqi. Comparison and analysis of dynamic characteristics of three typical floating wind turbines [J]. Acta Energiae Solaris Sinica, 2015, 36(12): 2874-2881 (in Chinese)

[12] 杨佳佳, 贺尔铭, 胡亚琪. 浮动平台内 TMD 对 Barge 式海上浮动风机的振动抑制研究 [ J ] . 西北工业大学学报, 2018, 36 (2) : 238-245

YANG Jiajia, HE Erming, HU Yaqi. Vibration mitigation of the Barge-type offshore wind turbine with a tuned mass damper on floating platform $[\mathrm{J}]$. Journal of Northwestern Polytechnical University, 2018, 36(2) : 238-245 (in Chinese)

[13] HU Y Q, HE E M. Active structural control of a floating wind turbine with a stroke-limited hybrid mass damper $[\mathrm{J}]$. Journal of Sound and Vibration, 2017, 410: 447-472

[ 14] YANG J J, HE E M. Coupled modeling and structural vibration control for floating offshore wind turbine[J]. Renewable Energy, 2020, 157: 678-694

[15] 刘国荣, 万百五. 一类非线性 MIMO 系统的直接自适应模糊鲁棒控制 [J]. 控制理论与应用, 2002, 19(5)：693-698

LIU Guorong, WAN Baiwu. Direct adaptive fuzzy robust control for a class of nonlinear MIMO systems [J]. Control Theory and Applications, 2002, 19(5): 693-698 (in Chinese) 


\title{
Application of adaptive fuzzy control to suppression vibration response of floating offshore wind turbine
}

\author{
YANG Jiajia, HE Erming, SHU Juncheng \\ ( School of Aeronautics, Northwestern Polytechnical University, Xi'an 710072, China)
}

\begin{abstract}
Floating offshore wind turbine is a complex rigid-flexible coupling nonlinear system, and the accurate dynamic model is difficultly established. Therefore, the wind-wave interference cannot be improved by adopting the conventional control strategy. In order to solve this problem, an adaptive fuzzy controller ( AFC) is used to suppress the dynamic response of floating wind turbine. Two correction factors are introduced to optimize the fuzzy rule, and the traditional fuzzy controller (FC) is firstly obtained. Since the balance positions change and structural parameter perturbation of the wind turbine, an AFC is designed and validated. Finally, the suppression vibration responses ability of floating offshore wind turbine by using the different control strategies is studied under the random windwave disturbance and blade pitch control system coupling effect. The simulation results show that the tracking ability of the AFC to the target value is obviously higher than that of the FC; Comparing with the passive control strategy, the suppression vibration effect on the power spectral density (PSD) of the platform pitch (PFPI) motion peak can increase by $39.06 \%$ by adopting the AFC.
\end{abstract}

Keywords : floating offshore wind turbine; vibration suppression; correction factor; fuzzy rule; adaptive fuzzy control

引用格式: 杨佳佳, 贺尔铭, 舒俊成. 自适应模糊控制在抑制海上漂浮式风力机振动响应中的应用 $[J]$. 西北工业大学学报, $2021,39(2): 241-248$

YANG Jiajia, HE Erming, SHU Juncheng. Application of adaptive fuzzy control to suppression vibration response of floating offshore wind turbine[J]. Journal of Northwestern Polytechnical University, 2021, 39(2): 241-248 (in Chinese) 